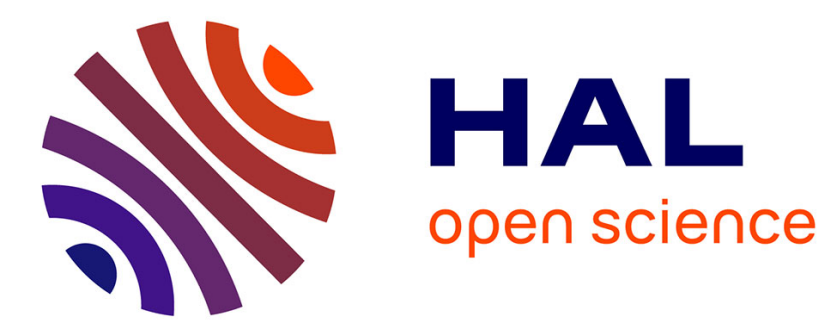

\title{
Propriétés magnétiques de NpSbTe et NpAsTe
}

\author{
A. Blaise, J.M. Collard, J.M. Fournier, J.P. Charvillat, A. Wojakowski
}

\section{To cite this version:}

A. Blaise, J.M. Collard, J.M. Fournier, J.P. Charvillat, A. Wojakowski. Propriétés magnétiques de NpSbTe et NpAsTe. Journal de Physique Lettres, 1984, 45 (11), pp.571-576. 10.1051/jphyslet:019840045011057100 . jpa-00232383

\section{HAL Id: jpa-00232383 https://hal.science/jpa-00232383}

Submitted on 1 Jan 1984

HAL is a multi-disciplinary open access archive for the deposit and dissemination of scientific research documents, whether they are published or not. The documents may come from teaching and research institutions in France or abroad, or from public or private research centers.
L'archive ouverte pluridisciplinaire HAL, est destinée au dépôt et à la diffusion de documents scientifiques de niveau recherche, publiés ou non, émanant des établissements d'enseignement et de recherche français ou étrangers, des laboratoires publics ou privés. 
Classification

Physics Abstracts

$75.60 \mathrm{E}$

\title{
Propriétés magnétiques de NpSbTe et NpAsTe
}

\author{
A. Blaise, J. M. Collard (*), J. M. Fournier (**), \\ Centre d'Etudes Nucléaires de Grenoble, Département de Recherche Fondamentale, \\ Section de Physique du Solide, 85X, 38041 Grenoble Cedex, France
}

J. P. Charvillat,

SAP - Etablissement de Marcoule, BP 170, 30200 Bagnols sur Cèze, France

\section{et A. Wojakowski}

Institut des Basses Températures et de Recherche Structurale, Académie Polonaise des Sciences, BP 937, 50-950 Wroclaw, Pologne

(Reçu le 25 novembre 1983, révisé le 29 mars 1984, accepté le 4 avril 1984)

\begin{abstract}
Résumé. - Les propriétés magnétiques de NpSbTe et NpAsTe ont été étudiées. NpSbTe reste paramagnétique jusqu'à $1,8 \mathrm{~K}$. NpAsTe devient ferromagnétique en dessous de $T_{\mathrm{c}}=25 \mathrm{~K}$; l'aimantation atteint $1,07 \mu_{\mathrm{B}} /$ atome de $\mathrm{Np}$ dans un champ magnétique de $40 \mathrm{kOe}$ et n'est pas encore saturée. Dans la zone paramagnétique, les résultats pour les deux composés s'accordent avec un état de valence +3 pour le $\mathrm{Np}$. Dans le cas de $\mathrm{NpAsTe}$, les résultats s'accordent également avec de récents calculs de champ cristallin, des mesures d'effet Mössbauer et des mesures de résistivité.

Abstract. - The magnetic properties of NpSbTe and NpAsTe have been studied. NpSbTe remains paramagnetic down to $1.8 \mathrm{~K}$. NpAsTe is ferromagnetic below $T_{c}=25 \mathrm{~K}$; the magnetization reaches $1.07 \mu_{\mathrm{B}} / \mathrm{Np}$ in a $40 \mathrm{kOe}$ field but is not yet saturated. In the paramagnetic range, results for both compounds indicate a $3+$ valence state of $\mathrm{Np}$ ions. In the case of NpAsTe, results are in agreement with recent crystal field calculations, Mössbauer effect and electrical resistivity measurements.
\end{abstract}

\section{Introduction.}

Les composés du type AnXY (avec $\mathrm{An}=\mathrm{U}, \mathrm{Np}, \mathrm{Pu} ; \mathrm{X}=\mathrm{As}, \mathrm{Sb}, \mathrm{Bi} ; \mathrm{Y}=\mathrm{S}, \mathrm{Se}$, Te) forment une famille homogène présentant des propriétés magnétiques intéressantes [1-5]. Ces composés cristallisent selon une structure quadratique de type anti-Fe ${ }_{2} \mathrm{As}\left(\mathrm{P} 4 / \mathrm{n} \mathrm{m} \mathrm{m} \mathrm{ou} \mathrm{D}_{4 \mathrm{~h}}^{7}\right)$ ou type UGeTe (I4/m m m ou $\left.D_{4 h}^{17}\right)$ [6-9].

(*) Boursier de Thèse de la C.C.E., Bruxelles, Belgique.

$\left.{ }^{* *}\right)$ Université Scientifique et Médicale de Grenoble, France. 
Seuls les composés d'uranium ont fait l'objet d'études systématiques concernant leurs propriétés physiques et leur structure magnétique [10-14].

En ce qui concerne les composés de neptunium et de plutonium, leurs structures cristallines ont été étudiées et précisées par Charvillat [9-15]. Les propriétés magnétiques des composés $\mathrm{NpAsY}(\mathrm{Y}=\mathrm{S}, \mathrm{Se}, \mathrm{Te})$ ont déjà été étudiées [5], mais une contamination de ces échantillons par du NpAs gênait considérablement l'interprétation des résultats et une nouvelle série de mesures s'avérait nécessaire.

\section{Méthodes expérimentales.}

La synthèse des composés a été effectuée au CEN de Fontenay-aux-Roses. Il s'agit d'une réaction solide-gaz entre des quantités stoechiométriques d'hydrure de neptunium et des non-métaux considérés, dans des tubes en quartz scellés sous vide secondaire [15]. On chauffe progressivement les ampoules à une température élevée $\left(760^{\circ} \mathrm{C}\right.$ pour $\mathrm{NpSbTe}$ et $850^{\circ} \mathrm{C}$ pour $\left.\mathrm{NpAsTe}\right)$ que l'on maintient ensuite pendant une à deux semaines. La caractérisation des échantillons se fait à l'aide de diffractogrammes X (méthode Debye-Scherrer, sensibilité relative : environ $5 \%$ ).

Les mesures de susceptibilité et d'aimantation ont été réalisées sur environ 100 à $200 \mathrm{mg}$ de poudre encapsulée dans un container étanche en aluminium de haute pureté. Les mesures de susceptibilité ont été effectuées entre $1,8 \mathrm{~K}$ et $300 \mathrm{~K}$ et pour des champs allant jusqu'à $17 \mathrm{kOe}$ à l'aide d'une classique balance de Faraday. Les mesures d'aimantation ont été effectuées à l'aide d'un magnétomètre à échantillon vibrant de type PAR 150 travaillant entre 4,2 K et $300 \mathrm{~K}$ et pour des champs allant jusqu'à $100 \mathrm{kOe}$. Dans le cas présent, les courants de Foucault induits dans le container en aluminium nous empêchent d'effectuer des mesures précises à basse température au-delà de $40 \mathrm{kOe}$.

Les mesures de résistivité ont été effectuées sur un échantillon de NpAsTe (aggloméré par pressage) suivant la méthode dite « 4 fils » en courant continu entre $4,2 \mathrm{~K}$ et $300 \mathrm{~K}$.

Des mesures d'effet Mössbauer ont été faites en champ nul, les résultats complets seront publiés prochainement [16].

\section{Résultats et discussion.}

3. $1 \mathrm{NpSbTe}$. - La structure cristalline de $\mathrm{NpSbTe}$ est du type anti-Fe $\mathrm{F}_{2} \mathrm{As}$ avec les paramètres de maille suivants : $a=4,330 \AA$ et $c=9,185 \AA$ [9]. La figure 1 représente l'inverse de la susceptibilité de NpSbTe en fonction de la température pour un champ $H=8200 \mathrm{Oe}$. Ces résultats s'accordent parfaitement avec une loi de Curie-Weiss du type $\chi=\frac{C}{T-\theta \mathrm{p}}$ où $\theta \mathrm{p}=-(25 \pm 1) \mathrm{K}$ et $C=(0,90 \pm 0,01)$ uem $/$ mole. Cette valeur de la constante de Curie nous permet d'estimer une valeur de $2,68 \mu_{\mathrm{B}}$ /atome de $\mathrm{Np}$ pour le moment effectif paramagnétique $\left(\mu_{\text {eff }}\right)$. Cette valeur est identique à la valeur théorique du moment effectif paramagnétique d'un ion libre $\mathrm{Np}^{3+}$ calculée dans le cas du couplage Russel-Saunders. (Dans le cas du couplage intermédiaire, $\mu_{\text {eff }}=2,83 \mu_{\mathrm{B}} /$ at. de Np.)

Le $\mathrm{NpSbTe}$ reste paramagnétique dans tout le domaine de température exploré $(1,8 \mathrm{~K}$ à $300 \mathrm{~K}$ ) et on n'observe pas d'anomalie de susceptibilité à basse température, liée à l'apparition d'ordre magnétique, malgré la valeur absolue élevée de $\theta \mathrm{p}$.

3. 2 NpAsTe. - La structure cristalline de $\mathrm{NpAsTe}$ est également du type anti-Fe ${ }_{2} \mathrm{As}$ avec les paramètres de maille suivants : $a=4,140 \AA$ et $c=8,909 \AA$. La figure 2 représente l'inverse de la susceptibilité de NpAsTe en fonction de la température pour un champ $H=8200 \mathrm{Oe}$. On observe sur cette courbe l'apparition d'un ordre ferromagnétique en-dessous d'environ $25 \mathrm{~K}$. On observe également un léger accident vers $80 \mathrm{~K}$.

Etant donné que d'une part les mesures d'effet Mössbauer n'indiquent qu'une seule transition vers $27 \mathrm{~K}$ [16] et que d'autre part, les courbes d'aimantation en dessous de $80 \mathrm{~K}$ montrent l'ap- 


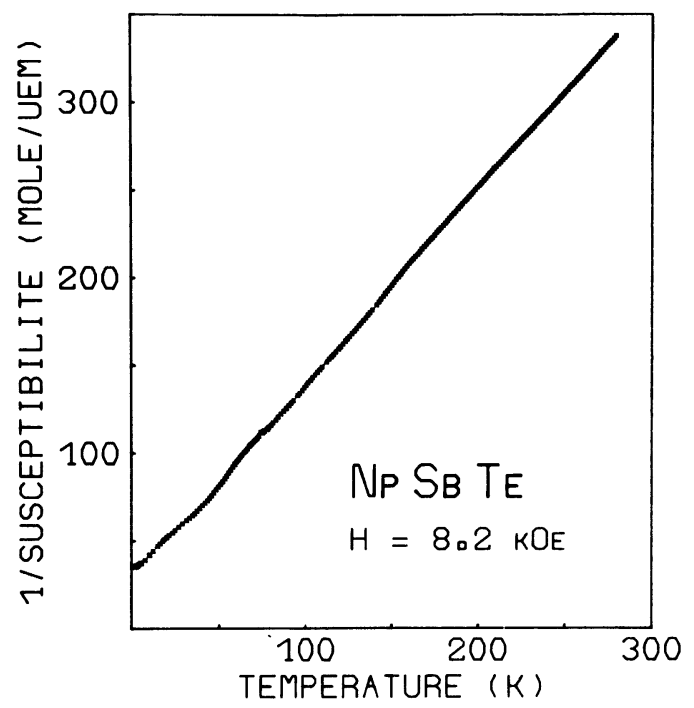

Fig. 1. - Inverse de la susceptibilité de NpSbTe en fonction de la température.

[Inverse susceptibility versus temperature for $\mathrm{NpSbTe}$.]

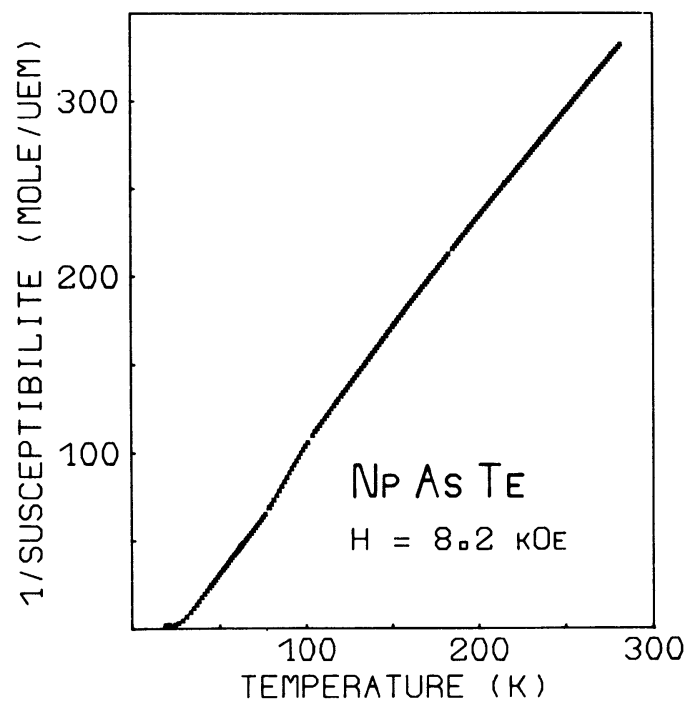

Fig. 2. - Inverse de la susceptibilité « expérimentale » de NpAsTe en fonction de la température (voir texte).

[Inverse « experimental » susceptibility versus temperature for $\mathrm{NpAsTe}$ (see text).]

parition d'une faible composante ferromagnétique, il est tout à fait probable que l'anomalie vers $80 \mathrm{~K}$ est due à une impureté ferromagnétique. Tenant compte des éléments introduits lors de la synthèse, nous attribuons cet accident à la présence d'un peu de $\mathrm{Np}_{3} \mathrm{As}_{4}$ dont la température de Curie $T_{\mathrm{c}}$ est justement égale à $81 \mathrm{~K}$ [17]. Partant des courbes d'aimantation isothermes 
à $51 \mathrm{~K}$ et $77 \mathrm{~K}$ et des mesures magnétiques effectuées sur $\mathrm{Np}_{3} \mathrm{As}_{4}$, nous pouvons dans un premier temps évaluer la proportion de $\mathrm{Np}_{3} \mathrm{As}_{4}$ à environ $0,25 \%$ en masse et ensuite corriger la courbe expérimentale de la figure 2 de l'influence de ce $\mathrm{Np}_{3} \mathrm{As}_{4}$.

On obtient alors la courbe illustrée par la figure 3. Celle-ci présente une concavité vers l'axe $T$ et on ne peut pas définir une valeur unique du moment effectif paramagnétique $\left(\mu_{\text {eff }}\right)$. La tangente à la courbe pour $T<50 \mathrm{~K}$ permet de définir un $\mu_{\text {eff }}$ à basse température égal à environ $2,35 \mu_{\mathrm{B}}$. De même, la tangente à la courbe pour $T>250 \mathrm{~K}$ permet de définir un $\mu_{\mathrm{eff}}$ à « haute température " de l'ordre de $2,6 \mu_{\mathrm{B}}$.

La figure 4 présente une courbe d'aimantation de NpAsTe en fonction de la température. Toutes les courbes réalisées présentent un point d'inflexion à $25 \mathrm{~K}$, valeur que nous pouvons donc attribuer à $T_{\mathrm{c}}$.

A $4,2 \mathrm{~K}$ et sous un champ $H=40 \mathrm{kOe}$, l'aimantation n'est pas saturée et atteint la valeur de $5950 \mathrm{uem} / \mathrm{mole}$, soit $1,07 \mu_{\mathrm{B}} /$ atome de $\mathrm{Np}$. La différence entre cette dernière valeur et la valeur de l'aimantation observée par effet Mössbauer $\left(1,6 \mu_{\mathrm{B}}\right.$ /atome de $\left.\mathrm{Np}\right)$ tient au fait que les mesures sont effectuées sur un échantillon polycristallin probablement anisotrope (structure quadratique).

Les résultats expérimentaux présentés ci-dessus sont en accord avec des calculs de champ cristallin présentés récemment par Amoretti et al. [18] concernant le NpAsTe dans l'hypothèse $\mathrm{Np}^{3+}$. La valeur du $\mu_{\text {eff }}$ observée à basse température $\left(2,35 \mu_{\mathrm{B}}\right)$ est voisine de celle calculée : $\mu_{\text {eff calc }}=2,12 \mu_{\mathrm{B}}$.

A haute température, le $\mu_{\text {eff }}$ expérimental $\left(2,6 \mu_{\mathrm{B}}\right)$ tend vers la valeur correspondant au peuplement de tout le multiplet $\left(2,68 \mu_{\mathrm{B}}\right.$ en couplage R.S.). Le splitting total a été estimé à environ $250 \mathrm{~K}$ dans la référence [18].

La figure 5 présente la résistivité de $\mathrm{NpAsTe}$ en fonction de la température. On note un maximum dans cette courbe situé vers $29 \mathrm{~K}$, valeur très proche de $T_{\mathrm{c}}$. Des résultats similaires ont été obtenus par A. Wojakowski lors d'une mesure de résistivité réalisée indépendamment sur une autre fraction de ce NpAsTe [19]. Cependant, ces mesures étant faites sur échantillon pulvérulent, elles ne peuvent avoir qu'une valeur indicative.

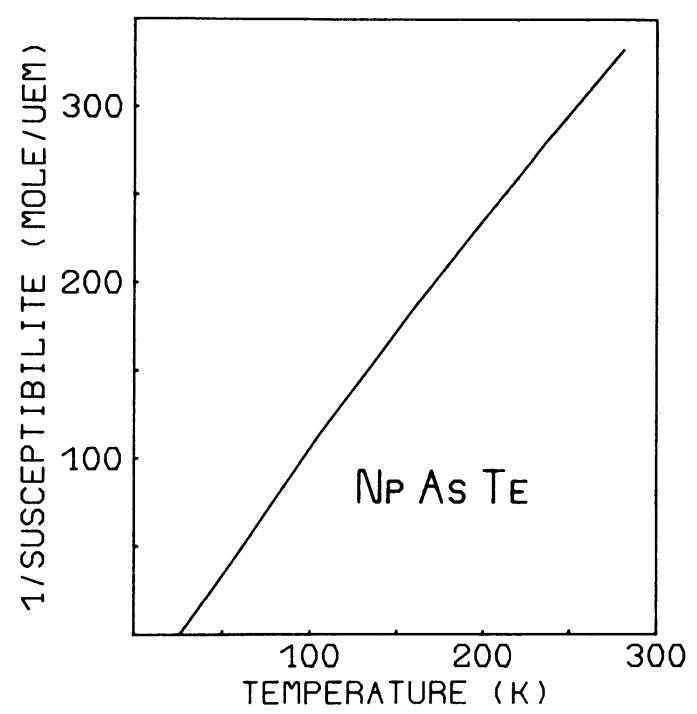

Fig. 3. - Inverse de la susceptibilité « corrigée » de NpAsTe en fonction de la température (voir texte). [Inverse « calculated » susceptibility versus temperature for NpAsTe (see text).] 


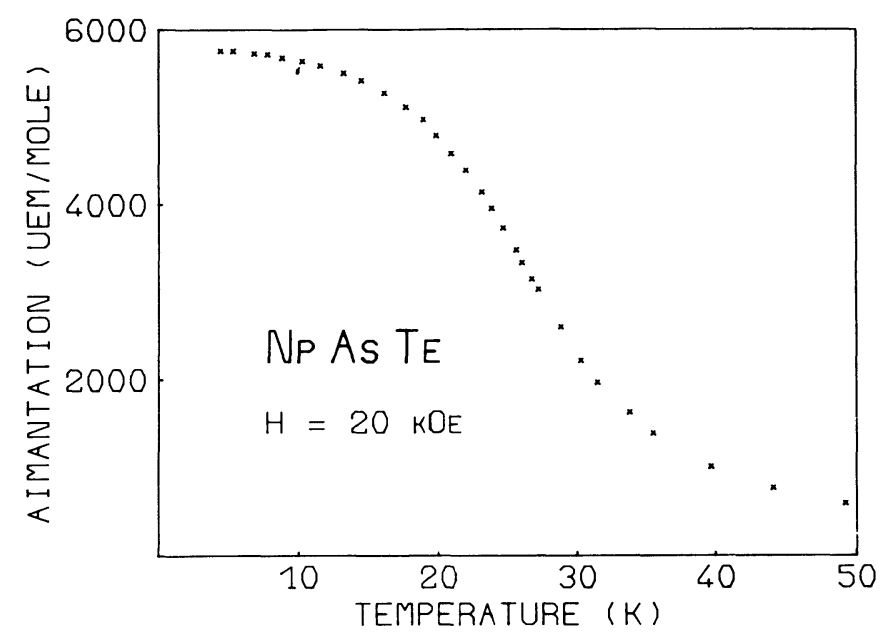

Fig. 4. - Aimantation de NpAsTe en fonction de la température.

[Magnetization versus temperature for NpAsTe.]

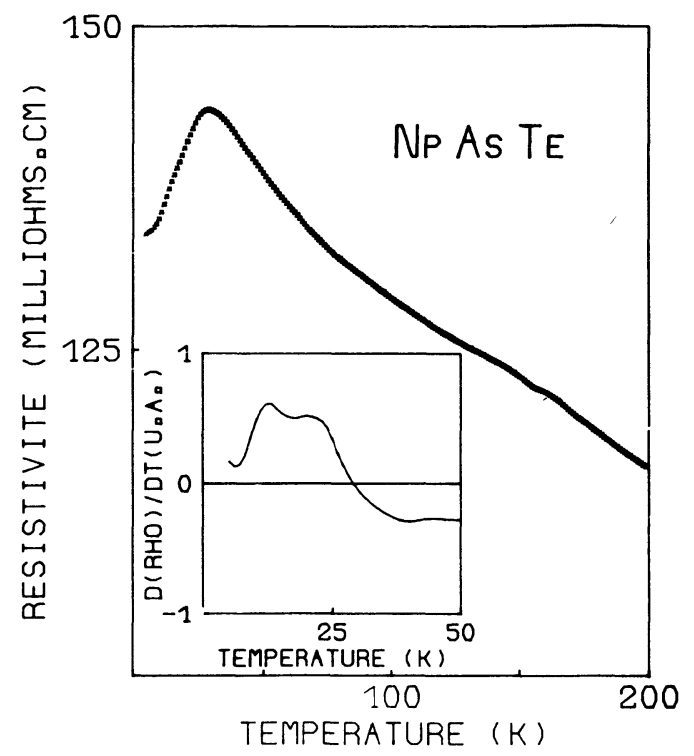

Fig. 5. - Résistivité électrique $(\rho)$ de NpAsTe en fonction de la température. En encadré : $\mathrm{d} \rho / \mathrm{d} T$ en fonction de la température au voisinage du maximum de résistivité.

[Electrical resistivity $(\rho)$ versus temperature for NpAsTe. Insert : $\mathrm{d} \rho / \mathrm{d} T$ versus temperature near $\rho_{\max } \cdot$ ] 


\section{Bibliographie}

[1] Hulliger, F., J. Less-Common Met. 16 (1968) 113.

[2] Zygmunt, A. and Duczmal, M., Phys. Status Solidi A 9 (1972) 659.

[3] Bazan, C. and Zygmunt, A., Phys. Status Solidi A 12 (1972) 649.

[4] Zygmunt, A. and Czopnix, A., Phys. Status Solidi A 18 (1973) 731.

[5] Blaise, A., Charvillat, J. P., Salmon, P., Wojakowski, A., Proc. 2nd Int. Conf. Electr. Str. Actin., Ossolineum-Wroclaw (Poland) 1976, p. 475.

[6] Pietraszko, D. and Lukaszewicz, K., Bull. Acad. Pol. Sci., Sér. Sci. Chim. XXIII (4) (1975) 337.

[7] Zygmunt, A., 11e Journées des Äctinides, Jesolo Lido, Italy (1981) p. 122.

[8] Flahaut, J., J. Solid State Chem. 9 (1974) 124.

[9] Charvillat, J. P., Wojakowski, A. and Damien, D., Proc. 2nd Int. Conf. Electr. Str. Actin, Ossolineum-Wroclaw (Poland) 1976, p. 469.

[10] Wojakowski, A., Henkie, Z. and Kletowski, Z., Phys. Status Solidi A 14 (1972) 517.

[11] Blaise, A., Lagnier, R., Wojakowski, A., Zygmunt, A. and Mortimer, M. J., J. Low Temp. Phys. 41 $(1 / 2)(1980) 61$.

[12] LeciejewicZ, J. and Zygmunt, A., Phys. Status Solidi A 13 (1972) 657.

[13] Zygmunt, A., Murasik, A., Ligenza, S. and Leciejewicz, J., Phys. Status Solidi A 22 (1974) 75.

[14] Zygmunt, A., Ligenza, S., Ptasiewicz, H. and Leciejewicz, J., Phys. Status Solidi A 25 (1974) K77.

[15] Charvillat, J. P., Thèse, Université de Clermont-Ferrand (France), 1977.

[16] Boge, M., Chappert, J., Wojakowski, A., A paraître.

[17] Blaise, A., Fournier, J. M., Roudault, E., Wojakowski, A., 12es Journées des Actinides, Orsay, France (1982) 20.

[18] Amoretti, G., Blaise, A. and Fournier, J. M., 13es Journées des Actinides, H1, Eilat, Israël (1983).

[19] Wojakowski, A., 13es Journées des Actinides, H4, Eilat, Israël (1983). 